SciCom meeting held in San Francisco in December 1996. The motivation of forming this working group was to better coordinate and promote all scientific activities on high-resolution late Quaternary paleoceanography of the western Pacific marginal seas (from the Bering to the Tasman Seas), and to better exchange information on paleoenvironmental and paleoclimatic records from the western Pacific covering the last 300,000 years.

The goal of this particular workshop was to establish a partnership that would significantly enhance data sharing and access, essential to the understanding of key issues of the western Pacific and Asian environmental variability. More specifically, the WEPAMA working group meeting was aimed at providing an important incentive to the development of future giant piston coring programs using the French vessel Marion Dufresne in the western Pacific marginal seas. Workshop participants included representatives of major members in the IMAGES program from the US, France, the Netherlands, Japan, Australia, Colombia, China, and Taiwan.

The meeting began with an introduction to the background of the IMAGES program, followed by discussions on scientific questions of the western Pacific paleoceanography: I. East Asia Paleomonsoons; II. Western Pacific Warm Pool Variability; III. SST and Circulation Patterns Since the Last Glaciation; IV. Land-Sea Paleoclimatic Linkage; and V. New Techniques and Proxies. The working group identified approximately 30 potentially good sites for giant piston coring in future IMAGES cruises in the year of 2001, and proposed the schedule for the next meeting.

\section{Chen-Tung Arthur Chen}

Institute of Marine Geology and Chemistry National Sun Yat-Sen University, Kaohsiung, Taiwan ctchen@mail.nsysu.edu.tw

\section{Min-Te Chen \\ Institute of Applied Geophysics \\ National Taiwan Ocean University, Keelung, Taiwan chenmtb212@ntou66.ntou.edu.tw}

\title{
Paleohydrology and Paleoclimate as Reflected in Lake-level Changes in China
}

\author{
Nanjing, China, 26-29 April, 1999
}

About 40 geologists, geomorphologists, chronologists, palynologists, paleoclimatologists, and paleo-GCM experts from China, Germany, Australia, the USA, and the UK attended this international workshop with support and sponsorship from the National Natural Science Foundation of China, the Chinese Academy of Science and the MaxPlanck-Institute for Biogeochemistry, Germany. The workshop was convened with the twin aims of building up the existing Chinese Lake Status Data Base (CLSDB), as a contribution to the International Global Lake Status Data Base records (GLSDB) program, and taking the first steps towards investigating the comparison of climate reconstruction based on Chinese lake records with climate model simulations. The effort is a contribution to the PMIP program, in which coupled-ocean simulations are being conducted by several groups, including scientists associated with the Testing Earth System Models with Paleoenvironmental Observations project (TEMPO).

The presentations and discussion began with reviews of recent advances in paleo-monsoon reconstructions mainly based on lake records, with "Recent research on the Asian paleomonsoon" (An Zhisheng), "High lake level records and enhanced Asian monsoon during 30-40 ka in Tibet" (Shi Yafeng), "Paleoclimate and Africa-Asian monsoon" (Sandy Harrison), "Paleomonsoon records from Australia" (Liz Picket). There were also several database demonstrations including "A range of paleo-data bases, in the PAGES PEP II region and the paleomonsoon" (John Dodson), "Potential Global Lake Status Data Base" (Philipp Hoelzmann), and "The state-of-the art of Chinese Lake Status Data Base" (Ge Yu). Modeling presentations included "Climate simulations, including Paleoclimate modelling for $6 \mathrm{ka}$ and $21 \mathrm{ka}$ " (Paul Valdes), "Paleohydrology modelling in Western China since 126 ka" (Mike Coe) and "PMIP-paleoclimate data and modeling comparisons" (Sandy Harrison).
There was a series of reports on regional lake sedimentology, paleohydrology and paleoclimate and demonstrations of ongoing lake research from the Tibetan Plateau and inland Xinjiang ( $\mathrm{Li}$ Shijie, Li Binyuan, Wang Fubao, Zheng Mianping, Tang Lingyu, Li Shengfeng), Inner Mongolian Plateau (Xiao Jule, Shen Ji), the Yunnan Plateau (Xue Bin, Zhang Zhenke) and eastern parts of China (Xu Qinhai, Yang Dayuan, Guo Shengqiao, Wang Jian, Xiao Jiayu). The lake sedimentary records presented were mostly from the Holocene but many lakes from western China have records extending beyond the last glacial maximum, to 30-40 ka BP (Figure 1). Numerous geomorphologic investigations of these lakes show evidence of high stands and absolute lake levels in the past. Changes in lake status have been reconstructed on the basis of changes in lithology, geochemistry, geobiology and archaeology. The chronology for changes in lake status is based on radiocarbon dates, thermo-luminescence dates, and paleomagnetic and stratigraphical correlations. All have produced compelling evidence for major climatic variability across eastern Asia's monsoonal regions. There were very high lake-levels in Tibet at the start of the period 30-40 ka BP, and we know that conditions on the eastern plains of China during the LGM were very dry. Many lake level changes are nearly synchronous over China during the midHolocene. A few of the talks dealt with changes in fluvial systems in China, such as the extremely large flood events recorded in the channel forms of the Huanghe River (Yellow River) in the Holocene. Bin Xue and Ge Yu also reported on their recent work on the CLSDB lake status coding and documentation procedures during the workshop.

The workshop culminated in a provisional agreement to continue work towards the establishment of CLSDB. One of the first goals for the CLSDB is to create a database of lake status records spanning the last 30,000 yr BP. Addi- 


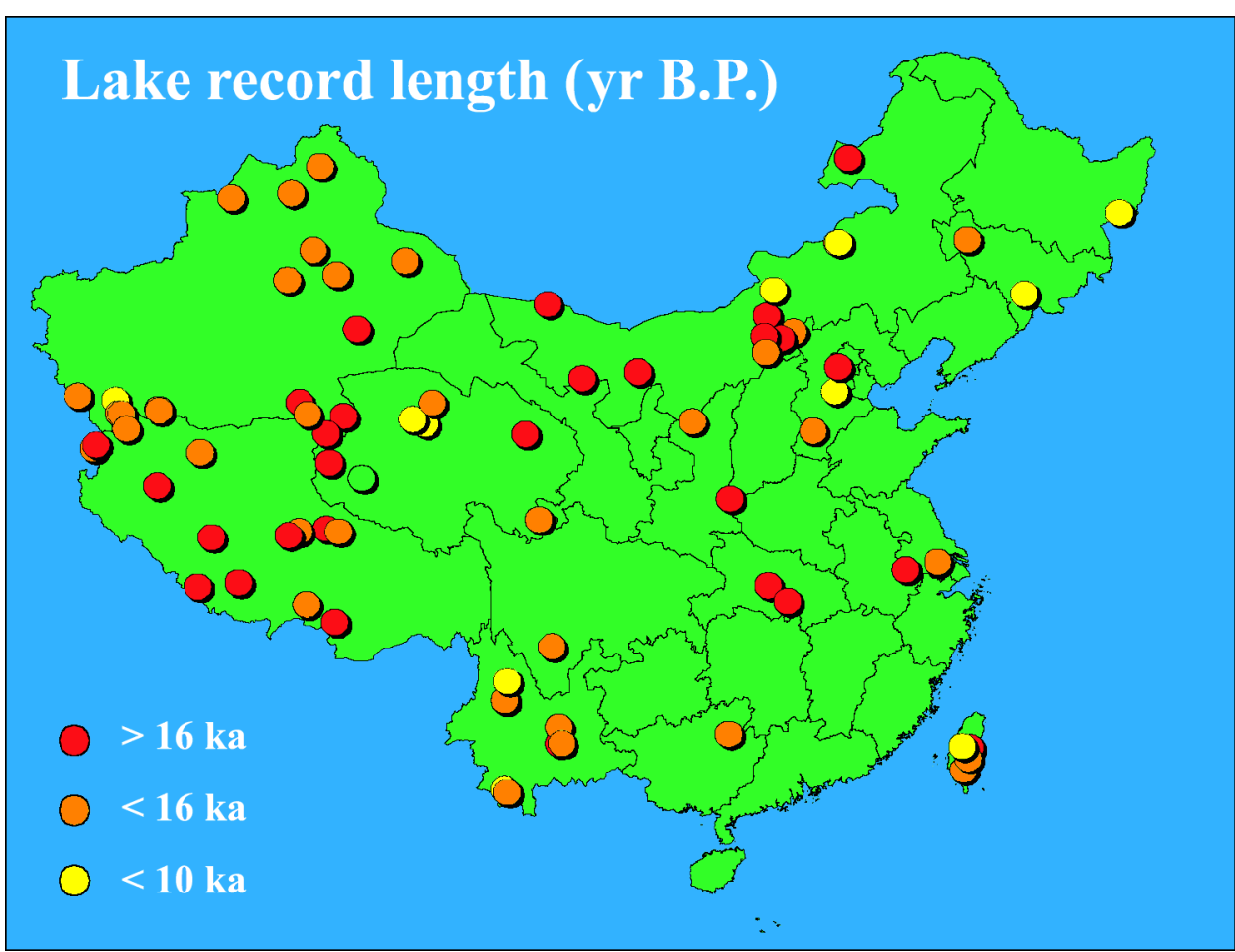

Figure 1: Record length (ka) of lake status from China Lake Status Data Base. Ca 35\% lakes have records spanning beyond the last glacial maximum, and ca 10\% lakes back to 30-40 ka BP.

tional information will also be included for model validation to make the database serve the Earth science community in general. A set of protocols on the CLSDB archiving and accession policy was agreed to during the workshop.

The presentations and discussion raised some key environmental questions:

- What have been the patterns of climate change (precipitation and evaporation) on millennial to century time-scales in Asian monsoon regions?

- What is the relative importance of external forcing (e.g. precessional orbital forcing) vs. internal mechanisms during the period 30-40 ka BP?

- What is the explanation for high lake-level during the LGM in the Tibetan Plateau region?

- What has been the interplay between the westerlies and monsoon systems in eastern and southern Asia?

- What have been the impacts of climate variations during the last millennia on fluvial dynamics in the large river systems of China?

These questions will motivate future data-modeling comparison studies to enhance the understanding of the dynamic mechanisms which underlie cli-

\section{VARVE 99}

\author{
Lammi, Finland, 13-17 ApriL, 1999
}

Interest in annually laminated sediments (i.e. varves) has expanded considerably as studies on short-term and rapid past environmental changes have intensified within global change study programs. The impetus to hold the VARVE 99 workshop originated at the PAGES SSC meeting at Pallanza, Italy in June 1998. The aims of the VARVE 99 workshop, held at Lammi, Jyväskylä and Espoo, and organized by the Geological Survey of Finland (GSF), were to introduce the assembly of field and laboratory methods currently in use in Finland, to give progress reports on ongoing projects, to exchange ideas, and learn of recent advances elsewhere. The participants came from Europe and North America. Several members of the European Lake Drilling Program (ELDP, sponsored by the European Science Foundation) attended the workshop and a meeting of the Northern Europe Regional Group of ELDP was also held. The workshop also stimulated interest within the tree ring community and plans for future co-operation (i.e. in EU funded research programs) were initiated. It is clear that studies of annually laminated sediments will play an increasing role in the PAGES activities.

The workshop was divided into practical field and laboratory sessions, and paper and poster sessions. Lake Korttajärvi in Jyväskylä, Central Finland was visited for a coring demonstration. A coring team from GSF took several long cores using a Kullenberg corer and a piston equipped gravity corer developed at GSF (Figure 1). Working on ice, a three person coring team was able to recover, in less than half an hour, a $10 \mathrm{~m}$ long core from beneath $17 \mathrm{~m}$ of water with the help of $2 \mathrm{~m}$ high tripod and a small engine-operated winch. $300 \mathrm{~kg}$ of lead weights were added to ensure continuous but slow penetration. In principle, water depth is not a limiting factor and up to $15 \mathrm{~m}$ long cores, perhaps longer, can be recovered. Slow penetration seems to produce unbroken sediment sequences, whereas cores taken by a Kullenberg-type gravity corer often contain discontinuities, which are obviously the result of sudden penetration. 